\title{
Voyage au bout de la nuit, un roman de la compassion démocratique ?
}

Si nous relisions Voyage au bout de la nuit à nouveaux frais ? Si nous envisagions que Céline, dans ce roman, se livre à une expérience de narration qui est aussi une mise à l'épreuve des valeurs déclarées de la démocratie et de la République ${ }^{1}$ ? Le laboratoire romanesque ne lui permet-il pas en effet de soumettre le personnage-narrateur, Bardamu, à une initiation au nom de laquelle on a pu rapprocher Voyage au bout de la nuit de Candide ${ }^{2}$, mais aussi à une émotion de l'humain ? C'est cette émotion que nous interrogerons ici, en proposant plus précisément pour hypothèse de lecture la clef de la compassion démocratique. Le roman célinien, avec ce qu'il doit aux romans populistes et ce qu'il corrige de leur esthétique et de leur éthique, occupe une place particulière, non seulement en vertu du parfum de scandale dont la suite des œuvres céliniennes allait l'affecter, mais surtout eu égard aux chances perdues et aux paris dédaignés qu'il re-présente à la conscience de ses lecteurs. Ces lecteurs, dans la mesure où le temps de la diégèse de la fin du livre coïncide avec le temps de l'écriture - et donc de la lecture-, sont aussi les concitoyens de son auteur.

\section{Le contexte historico-poétique du Vovage : le roman des années 30}

Voyage au bout de la nuit fait une irruption tonitruante sur la scène littéraire en 1932. Roman-cri contre la décadence, discrédité par le bellicisme, la colonisation, l'exploitation ouvrière, le taylorisme, la déshumanisation des banlieues, il ne s'inspire pas simplement de la

\footnotetext{
${ }^{1}$ Selon J.P. Martin, c'est le seul roman de Céline, les « autres » étant précisément des pamphlets (Contre Céline, Paris, José Corti, 1997).

${ }^{2}$ C'est ce que suggère M.C. Bellosta dans Céline ou l'art de la contradiction (Paris, PUF, 1990). A ceci près qu'il n'y a plus de jardin à cultiver à la fin du roman de Céline, mais des terrains soumis à la spéculation immobilière.
} 
thèse de l'essai de Spengler, Le Déclin de L'Occident (1918-1922). Il renvoie à Barbusse pour la critique du capitalisme et de la logique de guerre, Barbusse dont Céline a admiré Le Feu, publié en 1925. Il fait signe à Dabit : Céline s'adresse à Denoël parce qu'il a été édité Hôtel du Nord. On a d'ailleurs pu interpréter le choix onomastique du patronyme «Bardamu » à la juxtaposition des trois premières syllabes des noms de trois romanciers célébrés par Céline, le troisième étant Ramuz pour la présence de la parole du peuple et la connivence établie par le conteur avec le destinataire du livre. Or pour le romancier Céline, publier un livre-cri, au-delà du geste expressionniste, assigne le destinataire à se reconnaître en même temps comme le bénéficiaire de cette protestation enragée, à moins qu'il ne figure parmi les co-responsables de cette inhumaine condition humaine.

Le roman fut donc unanimement salué, ce qui établit sa force et sa faiblesse combinées. Il fut reçu globalement comme un livre de gauche, ce que justifient les thèmes de l'exploitation ouvrière dans les usines Ford à Détroit et de l'exploitation coloniale en Afrique, et la thèse de la défense des humbles. La critique ravageuse du capitalisme bourgeois et du militarisme qui mène au massacre explique qu'il fut encensé par Aragon. Mais il fut dénoncé par Nizan, plus sensible à l'ambivalence politique du roman : une ambivalence telle que l'extrême-droite s'y retrouva.

En effet Léon Daudet vit en Céline un nouveau Léon Bloy. Il reconnut la thèse de droite qu'est l'hygiénisme articulée au thème de la pourriture. L'inclination politique sensible dans l'anti-capitalisme, l'anti-militarisme, l'anti-bellicisme, l'anti-colonialisme - lui sembla compensée par la violence envers le relâchement et la vilénie du peuple.

Le roman fut en outre remarqué par Mauriac et Bernanos. Bernanos écrivit en 1932 un des premiers articles sur Voyage, sensible à la dimension métaphysique et à l'interrogation existentielle que Céline expliquait à un journaliste dès la parution du roman: "L'homme est nu, dépouillé de tout, même de sa foi en lui. C'est ça mon livre ». C'est cette dimension compassionnelle, appuyée sur le sentiment d'appartenance à une communauté malheureuse, qui retiendra notre attention ici. Sous la plume de François Mauriac et de Bernanos, la compassion s'éprouve du sein du christianisme. Bernanos s'incline devant le « visage sacré de la misère » mis à nu par le roman de Céline et discrimine deux catégories de lecteurs : ceux qui n'ont jamais vu ce visage et s'offusquent du roman, ceux qui le reconnaissent et répondent à l'appel de la pitié: « Nous plaignons ceux que le spectacle de la solitude du pauvre, de son effrayant exil, incite au désespoir plus qu'à la compassion - nous voulons dire à toutes les 
audaces, à toutes les colères, à toutes les fureurs de la compassion $»^{3}$. Cette pitié chrétienne n'est pas la source de la compassion célinienne. Il s'en explique dans sa réponse à une lettre perdue de François Mauriac, en janvier 1933 : «Que je vous exprime d'abord toute ma gratitude un peu émerveillée par un tel témoignage de bienveillance et de spirituelle sympathie. Rien cependant ne nous rapproche, rien ne peut nous rapprocher ; vous appartenez à une autre espèce, vous voyez d'autres gens, vous entendez d'autres voix. Pour moi, simplet, Dieu c'est un truc pour penser mieux à soi-même et pour ne pas penser aux hommes, pour déserter en somme superbement. Voyez combien je suis argileux et vulgaire». La pitié qui reste à Céline, tout argileux et vulgaire qu'il est, c'est ce que nous proposons d'appeler la pitié démocratique. Ecrire pour penser aux hommes, voici le programme annoncé par le romancier, programme moral et engagé par l'enragement de sa plume. Ne pas déserter, c'est assumer littérairement la disqualification d'un certain humanisme, d'un certain positivisme progressiste, c'est inventer un roman qui déborde le document social, historique et pédagogique dont les contemporains de Céline ne se dégagent pas toujours ${ }^{4}$.

Comment se situe Voyage au bout de la nuit dans le paysage littéraire des années 30 ? C'est-à-dire des années au cours desquelles, comme l'écrivit Thibaudet, le roman se « sécularise », entre dans «le vif du sujet» et dans l'arène des questions d'actualité, et s'engage au présent au nom du présent - et de l'avenir dont il accouchera -? Est-il juste de détecter en Céline un nouveau «réaliste » ou un «néo-naturaliste »? L'Hommage à Médan qu'il prononce en 1933 réfléchit aux formes et aux enjeux de la narration du réel dans la lignée de Zola, et il accrédite cette hypothèse. Mais pour examiner plus exactement la position singulière de Voyage au bout de la nuit parmi les essais romanesques d'écriture de l'histoire au présent, il faut en rappeler les diverses modalités.

Dans les années 30, après une bonne décennie d'occultation et de divertissement, les romans de la société s'ouvrent à l'évocation de la première guerre mondiale et de Verdun. Ainsi dans L'Eté 14 (1936) Martin du Gard réactualise la guerre dont on sent sourdre une nouvelle menace ${ }^{5}$. Invasion 14 de Maxence Van der Meersch paraît en 1935, tandis que Verdun et Prélude à Verdun occupent une place clef dans Les Hommes de bonne volonté de

\footnotetext{
${ }^{3}$ L'article de Bernanos, «Au bout de la nuit », publié dans Le Figaro du 13 décembre 1932, est reproduit dans Voyage au bout de la nuit, Soixante-dix critiques, 1932-1935, A. Derval (éd.), IMEC, 1993, rééd. 10/18, 2005.

${ }^{4}$ La mise en fiche et en ordre du réel cède parfois pourtant au soupçon dont parlait Stendhal. Le souffle du soupçon impose une nouvelle ère selon Nathalie Sarraute, laquelle commence à écrire Tropismes en 1932 précisément.

${ }_{5}^{5}$ R. Martin du Gard, auteur des Thibault qui parurent de 1922 à 1940, reçut le prix Nobel de Littérature en 1937.
} 
Jules Romains ${ }^{6}$. Mais Romains et Martin du Gard partagent encore un humanisme des Lumières et la foi en la puissance formatrice de la fiction, alors que le roman-cri auquel s'apparente Voyage au bout de la nuit est un roman critique: la guerre représente une fracture telle qu'on ne peut plus tenir un discours logique, à moins de tricherie sur le double plan épistémologique et éthique. Se substitue au logos le choix du pathos, le cri de l'âme, sorti des tripes avec lequel Céline composera un « roman de nerfs » ou d'émotion: "J'écris comme je sens", écrira-t-il à Léon Daudet à propos de Mort à crédit. D'où la dimension expressionniste du roman célinien, il grince et interpelle les sens et le coeur. Il est dit, non pas écrit ; vociféré, non pas ciselé. Puisqu'après cette première guerre mondiale, l'histoire ne peut plus être appréhendée que comme chaos, éprouvée qu'à travers le désordre des affects, c'est l'énonciation qui va communiquer ce désordre, le lancer à la face du ciel de façon à le faire entendre, et à le faire partager.

A côté de ces romans de la société, tout entière touchée par la guerre, les romans du peuple se concentrent sur un de ses éléments: le roman populiste, le roman prolétarien, le roman socialiste ont en commun le thème du peuple. Toutefois leurs choix esthétiques divergent.

Pour le roman populiste, le peuple est l'objet d'un discours pédagogique, clair, voire scolaire. En 1927 paraît un "Plaidoyer pour le naturalisme" de Thérive, qui veut revigorer le roman par un sursaut de réalisme. Le Manifeste du populisme en 1929 réactualise l'image stendhalienne du roman-miroir, dans l'idée que la présentation intelligible du réel éduquera le peuple. Mais aussi lui rendra hommage. Loin de l'esthétisme décadent, post-symboliste et loin de la psychologie de l'obscur (Proust), loin de l'immoralisme (Gide), le roman populiste développe l'optique morale de l'innocence dans une esthétique de la transparence: il s'agit d'en appeler à la vertu (celle de l'amour de la res publica, qui échappe à la corruption) dans une langue translucide. Les plus célèbres exemples sont Hôtel du nord (1930) et Faubourgs de Paris (1933) de Dabit, qui choisit un espace métonymique pour évoquer l'ensemble du peuple. Quand les sirènes se taisent raconte, en 1933, la fin de l'industrie. Van der Meersch y est proche de Zola, sans évidemment la lueur portée par le titre Germinal. Louis Guilloux est pour sa part lié au courant populiste dès La Maison du peuple en 1927. Mais on pourra surtout rapprocher Le Sang noir en 1935 de Voyage. Cripure y incarne la désillusion à l'égard de la Troisième République qui crut unifier la nation par l'enseignement et édifier le peuple par les valeurs républicaines : "Nous étions livrés aux professeurs, c'est-à-dire à la mort sous toutes

\footnotetext{
${ }^{6}$ Cette fresque restitue la vie «unanime» du 6 octobre 1908 au Front populaire de 1936 selon un procédé narratif simultanéiste. La parution s’étale de 1932 à 1946.
} 
ses formes". Reprenant à son tour la formule de Tolstoï, «la vérité de ce monde, c'est la mort », telle quelle dans Voyage, Guilloux la parachève en dénonçant particulièrement la mythologie du confort et du bonheur qui avilit les âmes et les abuse en les appâtant : "La vérité de ce monde, ce n'est pas qu'on meurt, c'est qu'on meurt volé".

Pour le roman prolétarien, le peuple est un objet et un enjeu non seulement social mais religieux. Poulaille théorise l'engagement de l'écrivain au service du peuple dont il nourrit une véritable mystique (cf Le Pain quotidien en 1931, qui sera suivi par Les Damnés de la terre en 1935, Pain de soldat en 1937).

Le roman socialiste, quant à lui, n'a pas encore adopté le réalisme socialiste, qui sera importé d'URSS en 1934. Mais deux noms l'illustrent déjà : Nizan et Aragon. Ce roman se subordonne à l'idéologie marxiste et à une praxis de parti. Nizan dans Antoine Bloyé, en 1933, fait la chronique d'une existence pauvre et banale de petit bourgeois sans conscience critique, discipliné, ployé/broyé sous et par le capitalisme ${ }^{7}$. La Conspiration, en 1938, roman salué par Sartre et par Camus, raconte la révolte de jeunes issus de la société bourgeoise contre laquelle ils complotent, mus par un romantisme du refus et par l'illusion lyrique de la révolution. Nizan quittera le parti en 1939, parvenu à la conclusion que ce parti «n'est pas des nôtres », pas plus que «n'était des leurs » Céline, dont il avait deviné les équivocités. Aragon, lui, suivra la ligne du parti et celle d'une esthétique «illusionniste » (à la Maupassant). Le cycle du Monde réel met en fiction des personnages, des événements et des questions historicoidéologiques, pour annoncer un âge « où des livres s'écriront pour des hommes pacifiques et maîtres de leur destin ». Ces romans restent unis au pacte réaliste et à une vision hégélienne de l'humanité ; l'esthétique du «mentir vrai » présente le monde selon les conventions de la vraisemblance, pour l'améliorer soit en dénonçant des abus et des erreurs, soit en magnifiant des options ou des paris.

A côté, un roman plus philosophique que pédagogique se développe, autour d'interrogations éthiques, métaphysiques aussi bien que politiques. C'est évidemment le cas de La Condition humaine de Malraux en 1933. Le questionnement spirituel qu'avait remarqué Mauriac dans Voyage au bout de la nuit n'autorise pas à identifier les réponses de Céline à celles de Malraux. Céline ignore «la fraternité virile », le "sacré en pitié » et le «sacré en néant $»^{8}$. Bardamu n'envisage pas le Mystère du Bien, tout enfermé qu'il est dans la nuit et la

\footnotetext{
${ }^{7}$ Nizan dénonça le pacte germano-soviétique en 1939, et tomba à Dunkerque en 1940.

${ }^{8}$ Des Noyers de l'Altenburg (1943) à Lazare (1974), Malraux revient sur l'épisode de la première utilisation des gaz par les Allemands contre le front russe, sur la Vistule. On y vit les soldats allemands revenir dans leurs lignes le dos chargé du corps des soldats russes, comme du poids de l'humanité tout entière. «L'assaut en pitié » de la Vistule est un moment qui joue « le rôle d'épiphanie » dans la pensée de Malraux.
} 
morbidité. L'intuition de la transcendance lui manque, à lui qui traverse l'épaisseur apocalytique de l'histoire sans pouvoir en épuiser la noirceur' ${ }^{9}$

Sartre, en revanche, emprunte à L'Eglise de Céline l'exergue de La Nausée en 1938 : « C'est un garçon sans importance collective, c'est tout juste un individu ». Dans ce vocable apparaît l'ambivalence des rapports qu'entretiennent, dans la démocratie, individualité et collectivité. Favorable selon les origines puisées dans le libéralisme au développement de l'individu, la démocratiee est-elle aussi propice, au nom de l'égalité, au sentiment de la communauté ? Faut-il être déjà un individu pour accéder à la dimension collective, ou bien la subjectivité s'accomplit-elle dans la communauté ? Par ailleurs, La Nausée dit la contingence de l'existence, le vertige d'être-là, et introduit la phénoménologie de Husserl et Heidegger dans le paysage littéraire français. Mais celle-ci ne fait pas oublier la donnée fondamentale issue de Kierkegaard qu'est l'angoisse. Or les existentialistes, marqués par l'angoisse qu'exhale Voyage au bout de la nuit, le lurent comme un roman phénoménologique de la mort. Car, s'en prenant aux phénomènes, aux symptômes, aux gestes, aux sensations de dégoût et de répulsion, il ne prononce aucune condamnation morale.

Cette proximité nauséeuse de Céline et de Sartre se poursuit sur le plan de la réflexion esthétique, sinon des propositions pratiques. La contestation de l'ordonnancement du roman qui restitue du sens là où il fait défaut nourrit le concept sartrien de « littérature de situation ». Donner l'illusion d'une nécessité, d'une signification et d'une orientation en racontant une vie au passé $\left(d^{\prime}\right)$ après sa fin, c'est lui conférer une intelligibilité en transformant les événements narrés en moments parfaits. Or cohérence, continuité, totalité construites par l'écriture romanesque sont des leurres, qu'il faut remplacer selon Sartre par une littérature qui dise la contingence de l'existence, l'aléatoire, la circonstance, et selon Céline par une littérature de l'oralité et de l'énonciation vive.

Pour récapituler, la famille la plus immédiate du Céline en 1932 est constituée des populistes. Mais ni l'espoir de Poulaille ni le sens de l'éducation n'étreignent Céline, dont l'interrogation et la fureur s'enracinent dans un terreau existentiel. Il ne connait non plus ni l'espérance malrucienne ni la confiance dans les discours de gauche. Céline occupe donc une place originale en ce qu'il se penche, à la faveur des révélations dues à la Grande Guerre, sur le peuple, sur la démission générale dont il fait les frais, sur le sens de la communauté démocratique: le peuple est le grand trompé de Voyage, la victime d'un mensonge collectif et

\footnotetext{
${ }^{9}$ Histoire qui commence justement à Noirceur-sur-la-Lys, nom romanesque d'un lieu inspiré du lieu réel Crèvecoeur-sur-l'Escaut.
} 
d'un leurre organisé. De ce point de vue, on peut considérer le roman anarchiste ${ }^{10}$ qu'est Voyage au bout de la nuit comme un roman de la pitié démocratique.

\section{L'horizon idéologique}

\section{Céline et la modernité}

Pour penser les termes de cette hypothèse de lecture, il faut envisager successivement les relations qu'entretient Céline avec la modernité, c'est-à-dire avec les philosophes des Lumières, avec la science et avec la psychanalyse.

D’abord, remontons à Rousseau dont la présence est aussi évidente que paradoxale. L'intertextualité rousseauiste s'élucidera si l'on discerne - de façon aussi peu réductrice que possible - le « Rousseau des littéraires » de celui « des philosophes », l'auteur des Confessions et des Rêveries et le penseur du Contrat social et du Second Discours sur les origines de l'inégalité. Les énoncés explicites d'anti-rousseauisme se dispersent dans l'oeuvre, mais selon un mode énonciatif paradoxalement emprunté à Jean-Jacques.

Evidemment la posture confessionnelle de Bardamu et le récit à la première personne exhibent à la fois l'analogie du mode énonciatif et le retournement de l'ethos. La confession de Bardamu est en effet celle d'un moi vil, sordide, voire délirant. « La grande fatigue de l'existence n'est peut-être en somme que cet énorme mal qu'on se donne pour demeurer vingt ans, quarante ans, davantage, raisonnable, pour ne pas être simplement, profondément soimême, c'est-à-dire immonde, atroce, absurde » (525). Les visiteuses de Lola à New-York se moquent de Bardamu à l'entendre ainsi se confesser à grands éclats et faire son petit JeanJacques devant elles » (274). Les retrouvailles de Bardamu et de Lola à New-York parodient celles de Jean-Jacques et de madame de Warens dans Les Confessions, la joie de l'une, indifférente à la pauvreté, s'inversant en déplaisir de l'autre, frappée par «l'abomination d'être pauvre ». Bardamu ignore enfin tout bon sentiment instinctif et semble condamné au ressentiment, puisque la tendresse de Molly ne le retient pas de confirmer jusqu'où peut s'étendre la nuit. Les rêvasseries du voyageur solitaire remplacent ironiquement les rêveries du promeneur solitaire. Molly prévient Bardamu: «Vous en êtes malade de votre désir d'en

\footnotetext{
${ }^{10}$ «Parlons-en de toi! T'es un anarchiste et puis c'est tout», dit à Bardamu Arthur Ganache dès le début du roman (Gallimard, coll. Folio, 1952, p. 17). Le soupçon émane ensuite des autorités militaires : «Les uns ont dit : " Ce garçon-là, c'est un anarchiste, on va donc le fusiller, c'est le moment, et tout de suite, y a pas à hésiter, faut pas lanterner, puisque c'est la guerre !..." » (p. 83). Dorénavant les références aux pages figureront entre parenthèses dans le corps de l'article.
} 
savoir toujours davantage [ ...] C'est le voyageur solitaire qui va le plus loin » (300). Le plus loin ne fera pas reculer la nuit, qui nous encercle infailliblement.

Autre retournement, les topoï de la nature sont des clichés rousseauistes inversés : « La Nature est une chose effrayante et même quand elle est fermement domestiquée, comme au Bois, elle donne encore une sorte d'angoisse aux véritables citadins. [...] Rien ne vaut le bois de Boulogne [...] pour faire affluer les souvenirs, incoercibles, [...] mélancolique et confidente inquiétude. [...] Ces arbres ont la douce ampleur et la force des grands rêves. Seulement des arbres, je m'en méfiais aussi depuis que j'étais passé par leurs embuscades » (76 - 78). C'est justement avant la première crise de délire devant le tir du Stand des nations que s'exprime l'anti-lyrisme bucolique.

Selon la même logique de l'antithèse qui gouverne déjà la posture éthique du narrateur, ce qui sous-tend la philanthropie et la pitié rousseauiste sous-tend a contrario la haine et le mépris de soi.

Dans la pensée de Rousseau, la pitié est un transcendantal affectif. Elle repose sur la notion du semblable, requiert une généralisation de l'amour de soi à l'amour d'autrui. Les autres fussent-ils absents, chacun peut les imaginer, en exerçant cette faculté que Kant a mise en avant dans la Critique de la Faculté de juger (§40). La compassion fondée sur la faculté d'imagination est donc universalisante à partir de la singularité d'un sujet. L'amour de soi, nécessaire à la préservation de la vie, mène à la pitié : «Je m'aime trop moi-même pour pouvoir haïr qui que ce soit », lit-on dans la sixième promenade des Rêveries du promeneur solitaire.

Or c'est bien à partir de l'affectivité que Céline construit sa vision anthropologique. Le fait et la fatalité d'être affecté par autrui s'incarnent en Bardamu du front de 14 à La GarenneRancy en passant par Petit Togo et Detroit. Mais à cet amour de soi qui conduit, selon Rousseau, à la compassion universelle, répond la haine de soi et la misanthropie qui en dérive:«Pendant la jeunesse, les plus arides indifférences, les plus cyniques mufleries, on arrive à leur trouver des excuses de lubies passionnelles et puis je ne sais quels signes d'un inexpert romantisme. Mais plus tard [...] on se rend compte, on est fixé, bien placé, pour comprendre toutes les saloperies que contient un passé. Il suffit en tout et pour tout de se contempler scrupuleusement soi-même et ce qu'on est devenu en fait d'immondices » (210).

Il y a bien, pour Céline comme pour Rousseau et Tocqueville, un raisonnement par généralisation analogique à l'universel, au lointain, à partir de soi et du semblable à soi. Mais la compassion rousseauiste semble d'abord se retourner en mortification. La généalogie de la pitié parait se renverser en généalogie de la haine et de la honte. L'imagination ne joue pas 
pour Bardamu de rôle constructif. Elle en est retenue par l'intuition de la négativité absolue. L'anti-rousseauisme de Céline s'inspire en effet du courant maurrassien, pour qui «Qui prolonge la double courbe romantique et révolutionnaire ouvre à l'Esprit une ample liberté de mourir » (Maurras, Romantisme et révolution). Dans la mouvance de la pensée de Maurras, contre Rousseau, contre la Réforme, contre la République, Bardamu pense la Révolution responsable de tous les désastres; elle a fait prendre en otage en priorité le peuple dont elle prétendait sauver les intérêts. La défense du peuple passe donc par l'accusation des Lumières.

Cette accusation-là réconcilie d'ailleurs les vues de Rousseau et celles de Céline. L'hypothèse philosophique selon laquelle la culture a échoué à rendre l'homme heureux est présentée comme un fait avéré par le roman de formation. La condamnation de la loi de l'enrichissement et du commerce, qui l'a de fait emporté dans la méthode de civilisation, se durcit lors du passage de Bardamu en Afrique coloniale, illustrant la mauvaise socialisation que déplore le Second Discours. Les institutions n'ont pas sauvé l'homme naturellement sensible de ce que la civilisation, la socialisation l'ont fait devenir, elles n'ont pas joué de rôle de compensation ni de protection.

En reprenant le Symbole qu'est Rome, Céline emprunte encore à la pensée de Rousseau. En moderne, il ne postule aucune normativité de la loi antique, mais il oppose et hiérarchise deux systèmes : celui de Rome, de l'autorité et de la vertu civique se convertit en système de contrainte sous la pression de la force : c'est celui auquel obéit Grappa qui «fouettait les soumis pour en extraire le tribut » (203). Mais le système Grappa est un moindre mal par rapport au «système Alcide, plus compliqué, dans lequel se discernaient déjà les signes du second stade civilisateur, la naissance dans chaque tirailleur d'un client, combinaison commercialo-militaire en somme, beaucoup plus moderne, plus hypocrite, la nôtre" (204).

Pour Céline en effet, la civilisation nourrit un délire, qui du premier au «second stade civilisateur » va s'aggravant à mesure que croît la catégorie de fous collectivement dangereux, ceux qui croient en la science et en la justice. Parapine le scientifique, aux yeux de Bardamu, incarne «une des rares formes redoutables de l'originalité, une de ces lubies aisément contagieuses : sociales et triomphantes pour tout dire [...] Ceux qui parlent de justice m'ont semblé, en définitive, être les plus enragés ! » (529). Mais qui a originellement mystifié les esprits et les peuples ? «Les philosophes, ce sont eux, notez-le encore pendant que nous y sommes, qui ont commencé par raconter des histoires au bon peuple... Lui qui ne connaissait que le catéchisme ! Ils se sont mis, proclamèrent-ils, à l'éduquer... Ah ! ils en avaient des vérités à lui révéler ! [...] C'est ça, qu'il a commencé par dire, le bon peuple, c'est bien ça ! C'est tout à fait ça! Mourons pour ça! Il ne demande jamais qu'à mourir le peuple ! Il est 
ainsi. "Vive Diderot !" qu'ils ont gueulé et puis "Bravo Voltaire !" En voilà au moins des philosophes! Et vive aussi Carnot qui organise si bien les victoires !» (92). Ceux que torture la marotte de la civilisation" (527) sont ainsi des mystiques fous et des "émancipés frénétiques" : l'oxymore de cette expression relève par la violence du propos la démence dont on parle.

Dans la même mouvance, s'attise la haine de l'intellectualité, trop futile ${ }^{11}$, de l'Ecole qui déresponsabilise, qui aliène et qui corrompt: "Les blancs, perfectionnés par l'Instruction publique, ils marchent tout seuls" (183). Vers quoi marchent-ils avec une telle bonne conscience? vers l'aliénation colonialiste et l'exploitation des populations africaines. Alors la politesse, la civilité, la délicatesse, le tact, la bonne tenue sont définitivement désuètes dans un monde avachi en pleine déliquescence, un monde débraillé, effiloché (254), dissous (262), déliquescent, débordant, dégoulinant, dégoûtant, dégueulasse, gâteux, baveux ${ }^{12}$.

A moins que la civilité et le débraillé ne soient les deux faces d'une même culture ? L'Amérique par exemple confirme la règle par excès de pudeur, d'ordre et de contrainte, mais aussi par la double contrainte puisque c'est le pays du comble de l'aliénation. En témoigne le contraste à New-York entre l'attitude des citoyens en surface et leur comportement dans les souterrains. La débandade y est perçue par les yeux éberlués de Bardamu. Cette débabdade n'est pourtant rien par rapport à la déshumanisation dénoncée lors du passage du « héros » dans les usines Ford à Détroit. Les abus de l'industrialisation, le taylorisme rappellent les scènes déchaînées de Charlot travaillant à la chaîne dans Les Temps modernes (1936) : la mécanisation des hommes est appelée par leur crainte de se faire remplacer par des machines. «On en devenait machine aussi soi-même à force et de toute sa viande encore tremblotante dans ce bruit de rage énorme qui vous prenant le dedans et le tour de la tête et plus bas vous agitant les tripes » (287). Car on l'affiche clairement, l'éducation de l'intelligence par le savoir et par le savoir-faire était un leurre : "C'est de chimpanzés dont nous avons besoin. $\mathrm{Ne}$ nous parlez plus jamais de votre intelligence" (idem). Le chimpanzé figure 1'homo economicus, l'animalisation de l'humain produite par le développement économico-social. Il s'oppose à l'asticot (426) qui, lui, métaphorise le "sous-homme claudiquant" (525), métaphysique, dont se souviendra Beckett dès la trilogie de Molloy. La République a inventé les «soldats gratuits, héros pour tout le monde et singes parlants» (16), sacrifiant à la majorité d'intérêt une minorité déclarée indigne de sorte que ce massacre n'indigne plus

\footnotetext{
${ }^{11}$ « [Princhard] il avait le vice des intellectuels, il était futile» (p. 94)

${ }^{12}$ On trouve ici une lecture apocalyptique du catalogue des attributs de la civilisation occidentale par différence avec la Kultur allemande, selon Norbert Elias (Über den Prozess der Zivilisation, 1939).
} 
personne. Même, la « roublardise immense » (69) du bellicisme et du militarisme convainc le peuple qu' « engraisser les sillons du laboureur anonyme c'est le véritable avenir du véritable soldat » $(91)$, réduit à de la chair à canon, à de la « viande saignante et tremblotante ».

Cet argumentaire pervers articule le dédain républicain pour la communauté populaire et la culpabilité chrétienne dont la République a aussi échoué à débarrasser le peuple. Loin que la philosophie ait remplacé au moins le catéchisme, elle l'a recouvert d'un discours de surface qui n'interdit aucunement l'efficacité de la foi en la faute. Il faut bien en effet pour tâcher de comprendre (puisque le démon de comprendre s'est emparé de la conscience populaire) que le pauvre ait à expier une faute immémoriale, celle de déranger le confort de la communauté. « Ils étaient coupables et c'était déjà bien gentil qu'on leur donne ainsi en souffrant l'occasion d'expier leurs indignités », croyait la mère de Ferdinand (125-126). «L'esclave doit être coûte que coûte un peu et même beaucoup méprisable. Un ensemble de petites tares chroniques morales et physiques justifie le sort qui l'accable. [...] Raté, débouché, dévoyé, dévoué, tout s'expliquait » (538). Le pauvre est donc le bouc émissaire justement sacrifié sur l'autel de la bourgeoisie libérale et capitaliste qui se déballera sur l'Amiral Bragueton, selon la logique du pharmakos, pour resserrer les liens de la communauté. Sous la satire sociale et la rage politique sonne encore l'accent du désespoir : «le déshonneur automatique et la honte inexpiable» sont $d u s$ parce que l'état de pauvre «indique en lui-même une indignité capitale, et ensuite parce que son acte comporte une sorte de tacite reproche envers la communauté »(90). Au-delà des invectives aux fauteurs de troubles, il y a l'immense compassion de Céline pour les «pauvres, les gens dont la mort n'intéresse personne » (69), les chimpanzés et les asticots, les humbles et les déshérités que côtoie Bardamu, pour les morts et pour les rescapés définitivement abrutis par la guerre parmi lesquels il se compte: «nous les viandes, destinées au sacrifice » $(127)^{13}$ formons une communauté de l'être pourla-mort, comme si la faute était «le péché éternel, irrémédiable et inexpiable d'être né » ${ }^{14}$.

Toute l'idéologie de la modernité et de l'Aufklärung est donc bousculée puisque « moderne » signifie aliénant, exploiteur, belliqueux. Céline, finalement, pousse à l'excès la pensée rousseauiste, jusqu'à la « sociophobie ». Le peuple corrompu n'est pas vertueux, il ne « comprend que l'argent et le théâtre ». Ainsi « ces semblables et monstrueuses inconsistances

\footnotetext{
${ }^{13}$ Le sacrificateur est figuré en Pinçon, « les gradés, les petits surtout, plus abrutis, plus mesquins et plus haineux encore que d'habitude », qui enragent « d'envoyer crever les autres qui n'avaient pas de carte » (41). Il est aussi incarné par le commandant du navire : «j’étais la bête, ils se faisaient déjà la main les sacrificateurs » (155).

${ }^{14}$ S. Beckett, Proust, 1931.
} 
sont bien faites pour dégoûter le plus patient, le plus tenace des sociophiles » (110). La foi de Voltaire dans la vertu du processus de civilisation est un leurre que révèle l'épisode cauchemardesque de la navigation de Bardamu à bord de L'Amiral Bragueton: "Nous vîmes à fleur de peau venir s'étaler l'angoissante nature des blancs, provoquée, libérée, bien débraillée enfin, leur vraie nature, comme à la guerre. [...] C'est alors que l'on se déboutonne éperdument et que la saloperie triomphe" (149). Céline proteste donc plutôt contre le culte voltairien de la modernité que contre Rousseau, qui sait lui-même, en philosophe moderne, que sont abolies la vertu et la Cité.

Mais, là où Rousseau voit dans l'Histoire un processus aléatoire, contingent, atéléologique, indéterminé donc perfectible, corrigible par une bonne socialisation, celle que postule Le Contrat social, Céline ne nourrit aucun espoir de réconciliation de l'individu avec la société. L'amour de soi ne résiste pas à ce qu'est l'homme devenu. Comme cet état de corruption semble devenu la seconde nature, la compassion pour ce qu'on a fait de l'homme ne déborde pas sur la pitié qui pourrait fonder une communauté. Faute de rationalisation sur la base de l'affectif, Voyage au bout de la nuit ne se propose pas comme un roman politique. L'anarchisme de Bardamu suspend encore la tentation du retour à l'ordre. Mais ce retour, on le pressent, sera fatal. Car c'est au nom la compassion, au nom de l'idéal démocratique, que Céline fait le procès de la République. Si aucune réparation ne peut s'envisager dans ce cadre, si la République porte la faute irrémédiable de ses mensonges et de ses impostures, ni la compassion démocratique, ni la philanthropie du médecin des misérables qu'est Bardamu n'empêcheront la sensibilité naturelle de dévier vers une négativité absolue. Car le sentiment de déréliction fondamentale rend problématique toute possibilité de réciprocité et de mutualité. Le roman ne permet donc pas de penser quelque re-médiation, quelque institution que ce soit qui permît de fonder sur le sens commun de la justice une communauté ni $a$ fortiori une constitution.

Qu'est-ce qui reste possible, et surtout impératif pour le romancier qu'est Céline ? Au moins de prendre le peuple au sérieux, sans en faire un enjeu mystique, politique ni électoral, c'est-à-dire de l'écouter, de lui donner la parole. C'est précisément parce qu'il a été envoyé sur cet «abattoir international en folie », victime d'une conspiration économico-polticomilitaire, que le peuple mérite absolument cette délégation. Céline se sert de sa propre expérience du front, qu'il projette dans la fiction de Bardamu, pour appréhender les troubles dus à ce nouveau visage de la guerre de masse « démocratique ». La question de la guerre et de ses conséquences psychiques et socio-politiques désastreuses se pose à la lumière des hypothèses de Freud et de leur influence sur le docteur Destouches. Transposée dans le roman 
de Louis-Ferdinand Céline, l'ultime élément de la modernité, la science de la psyché, se voit accorder une place capitale dans la narration et une fonction malicieuse dans la diégèse.

\section{Psychiatrie contre psychanalyse}

Ferdinand Destouches fit l'expérience de la psychiatrie pendant la première guerre et la découverte de l'oeuvre de Freud dans les années 2O, plus particulièrement des Considérations actuelles sur la guerre commencé en 1915 et d'Au-delà du principe de plaisir (1920). Céline fait allusion à l'hypothèse de l'inconscient en se moquant de la tendance de Bardamu à «moraliser» à tout prix dans de multiples sentences à la La Bruyère: "Tout l'inconscient se débine devant vous dès qu'on s'approche" (499). Il choisit pour narrateur un personnage marqué par la névrose traumatique de guerre, habité par une pulsion de mort qui, si elle ne recoupe pas le concept freudien, en garde la désignation ${ }^{15}$. Il accrédite $1^{\text {'hypothèse }}$ de la pulsion de mort et les analyses freudiennes de la névrose traumatique de guerre et du conflit intra-narcissique ${ }^{16}$.

Dans Voyage, la psychanalyse est évoquée de biais. C'est la psychiatrie, dans l'épisode initial de l'hôpital militaire de Kremlin-Bicêtre et dans l'épisode final de l'asile psychiatrique de Vigny-sur-Seine, qui a pignon sur rue. Pignon d'où s'observent les effets de la pensée psychanalytique.

Le psychiatre représente le scientifique fasciné par les progrès de la technique et des appareils, en soi potentiellement dangereux. Baryton qui dirige l'asile de Vigny-sur-Seine est finalement assimilé au "bouffon de chercheur" (360). Il incarne le positivisme, s'en tient aux faits, au corps, à la vessie, à la pourriture. De plus, il est menacé par la compétition libérale, concurrencé par d'autres instituts, il doit se moderniser pour satisfaire ses patients qui « insatiables n'en finissaient pas d'exiger, de réclamer toujours plus ». Le capitalisme traque donc Baryton comme il traque les immigrants à Détroit et les nègres à Topo. Mais en bon scientiste, il confirme le dogme rationaliste : "Je suis une bête à testicules, moi, et lorsque je tiens un fait j'ai bien du mal à le lâcher" (535).

La psychanalyse, elle, représente l'adversaire de cette religion de la science. Pour les psychiatres, elle se confond avec les forces du mal, celles qui menacent depuis 1900 la

\footnotetext{
${ }^{15}$ Marie Bonaparte encouragea d'ailleurs Freud à lire ce roman qui fictionnalisait l'instinct de mort étudié dans Au-delà du principe de plaisir.

${ }^{16}$ Voir infra. "La fin vers laquelle tend toute vie est la mort». Freud associa à la libido narcissique ces « instincts du moi » ayant pour mission de seconder «la vie dans sa course à la mort»: impénétrabilité à la représentation de notre propre mort, souhait de mort à l'adresse de l'étranger et de l'ennemi, ambivalence dans le deuil à l'égard de la personne aimée.
} 
civilisation et "nous précipite aux abymes" (533). C'est une "mode obscène", un scandale pour la pensée, pour l'ordre et la mesure qui sont les clefs du cartésianisme. "L'outrance étrangère" (534) résume la "mode de délirer sous prétexte de mieux guérir, mode obscène comme presque tout ce qui nous vient de l'étranger" (532). Baryton se fait un devoir de résister à l'influence pernicieuse, infectieuse et contagieuse de l'étranger, du désordre : la métaphore filée de l'épidémie suggère l'invasion inquiétante de la mode viennoise et la menace qui pèse sur l'idiosyncrasie nationale et l'identité culturelle. Rappelons que la première question du prologue porte sur l'existence de la nation française. La question de l'identité est donc absolument centrale. Céline est partagé entre deux cultes qui se contrarient : celui, barrésien, de l'exception française, et celui de l'énergie collective qu'incarnent des Allemands, des Russes ou des Juifs et qui manque à la France. Au nom de l'identité nationale, vulnérable par défaut de cette énergie, Baryton s'en prend à ce qui vient d'ailleurs. Jamais la judéité ni la germanité de Freud ne sont explicitées. Mais les allusions sont claires, car Freud postule le comble, c'est-à-dire l'étrange(r) au coeur de soi-même, en l'espèce, l'inconscient. La psychanalyse est ainsi dénoncée par Baryton comme une grave crise des mentalités qui pervertit, corrompt, liquéfie les esprits: "à coup d'analyses superconscientes" (533), "à force de se sublimer... dans la cave aux damnés... à force de se masturber la jugeote jour après nuit", "se forniquer à longueur de rêves", "ce n'est plus autour d'eux qu'une ragouillasse dégueulasse de débris organiques, une marmelade de symptômes de délires en compote qui leur suintent et leur dégoulinent de partout" (534) ${ }^{17}$.

L'option rationaliste domine le roman si l'on considère la proportion du volume discursif attribuée aux psychiatres. Pourtant, elle est habilement discréditée par deux procédés. D’une part l'énonciateur des discours sur la psychanalyse, Baryton, ratiocine plus qu'il ne raisonne. Il développe une hypertrophie de l'intelligence cartésienne et dialectique apparentée à une manie, voire à une névrose: ses "controverses sous-intellectuelles", ses "résolutions contradictoires", ses "propositions et réticences minuscules", ses "ergotages", forment une maladie qui risque de contaminer Bardamu. Son disciple ne pouvait que devenir dissident, car « il [1]'ensorcelait de sottises » (541-542). Le « cas Baryton » exemplifie donc le précepte anti-positiviste du roman : le «délire scientifique plus raisonné et plus froid que les

\footnotetext{
${ }^{17}$ Cette "Grande pagaille spirituelle" est mise au compte de cette science nouvelle, moderne, cette mode de l'intuition (mot qui appartient à Bergson, dans sa condamnation de la perte positiviste de l'âme) reprise par la psychanalyse), mode de l'impression, c'est-à-dire mode féminine, voire invertie qui dévirilise la société française: "nous voici en plein déluge! Ah on s'ennuyait paraît-il dans le conscient! On ne s'ennuiera plus! On a commencé par s'enculer, pour changer... Et alors on s'est mis du coup à les éprouver... les "intuitions" et les "impressions"... Comme les femmes!"(535).
} 
autres est en même temps le moins tolérable d'entre tous » (357). Sa phobie de la psychanalyse est la meilleure défense de la science freudienne.

D'autre part, la rivalité idéologique entre psychiatrie et psychanalyse n'est pas seulement thématisée, elle opère une fonction dramatique dans l'intrigue romanesque. Le discours de ces «bouffons de psychiatres » est dédit par l'évolution de Baryton en personne et par le déroulement de l'intrigue fictionnelle. La «bête à testicules » est en effet rattrapée par ce dont elle se défend, c'est-à-dire « l'autre » jusqu'à être " contaminée par la méditation », la « débâcle spirituelle ». Peu importe que ce soit l'apprentissage de l'anglais et le charme d'Albion qui aient raison de sa « raison ». L'essentiel, du point de vue de la polémique entre psychiatrie et psychanalyse, c'est que l'aliéniste aliéné « abjure » finalement sa foi (550). Ce geste et ce vocable réduisent la rationalité à l'objet d'un croire et non d'un penser. Ils la démythifient donc en la ramenant à un mythe fondateur de la modernité en passe d'être dépassée. Parapine à propos d'un assistant se moquait déjà de la ferveur équivoque de la dévotion scientifique : «Voici trente ans bientôt qu'à balayer mes ordures il n'entend parler autour de lui que de science. [...] Cependant loin d'en être dégoûté la moindre de mes singeries l'enivre! N'en va-t-il pas d'ailleurs de même dans toutes les religions? » $(361)^{18}$.

C'est donc la scienceen vigueur, la psychiatrie, qui sert de référence à l'aune de laquelle est évaluée la différence de la psychanalyse. Mais la malice du roman est remarquable, et le point de vue d'où l'on parle de la pathologie n'est pas le point de vue supporté par la voix narrative de Voyage au bout de la nuit.

En effet l'instinct de mort et la névrose traumatique de guerre sont des hypothèses $\mathrm{du}$ roman. Le personnage-narrateur Bardamu est aussi une figuration de l'auteur Destouches, médecin et soldat, dont le point de vue est proposé au lecteur. A la lecture, c'est en effet à cette voix et à cette vision que nous nous identifions. Or elles accréditent l'hypothèse freudienne du conflit intrasystémique du moi suscité par la nouvelle situation de guerre guerre de masse, « démocratique »- à partir duquel Freud fut amené à élaborer le concept de pulsion de mort ${ }^{19}$.

\footnotetext{
${ }^{18}$ Parapine représente Elie Metchnikoff, biologiste russe qui a publié en 1901 L'immunité dans les maladies infectieuses, en 1903 Etudes sur la nature humaine et Essais optimistes en 1907. Il reçut le Prix Nobel en 1908. Lors de la typhoïde de Bébert dans la fiction, Bardamu convoque Parapine qui, à l'instar de son modèle, passe de l'institut Bioduret à l'asile psychiatrique. Metchnikoff s'intéressa à l'instinct de mort et souligna que s'il existe, il n'a pas le temps de se manifester, car notre mort n'est presque jamais naturelle

${ }^{19}$ Dans Considérations actuelles sur la guerre en 1915, Freud aborde deux questions : celle de la désillusion d'après-guerre à l'égard de la « civilisation » de surface défaite par la brutalité belliqueuse des états, puis celle de la relation de l'homme à la mort. Il y revient précisément en 1932 dans la Lettre de réponse à Einstein « Le but de la vie est-il la mort?» La guerre annule la contradiction entre l'inconscient désir de tuer l'autre et l'impossibilité de se représenter soi-même mourant. Elle ne permet plus de penser la mort comme accidentelle.
} 
D'abord, la pulsion de mort dans Voyage au bout de la nuit est une transposition romanesque. Elle s'y exprime à travers «la vocation de meurtre » que Bardamu repère en Robinson. Robinson actualise une tendance radicale à la dislocation qui semble "plutôt somme toute comme une espèce de progrès sur ce qu['ïl]avai[ $\mathrm{t}$ ] observé jusqu'alors parmi les autres gens, toujours mi-haineux, mi-bienveillants » (390). Tous les gens, « quand ils sont debout, pensent à vous tuer » (388), mais la pensée dans l'état de paix reste virtuelle. La guerre, elle, a définitivement perturbé et déréglé le fonctionnement psychique, c'est-à-dire qu'elle a révélé les virtualités les plus barbares de l'esprit humain. D'ailleurs il n'existe pas $\mathrm{d}^{\prime}$ « autres véritables réalisations de nos profonds tempéraments que la guerre et la maladie, ces deux infinis du cauchemar»(525). C'est l'instinct de mort qui anime les créatures, à travers un immense narcissisme sado-masochiste. Bardamu se rend finalement à cette intuition de son double Robinson : «C'est tuer et se tuer qu'ils voulaient, pas d'un seul coup bien sûr, mais petit à petit comme Robinson avec tout ce qu'ils trouvaient, des vieux chagrins, des nouvelles misères, des haines encore sans nom quand ça n'est pas la guerre toute crue » (344).

La querelle idéologique entre psychiatrie et psychanalyse est surtout cristallisée autour de la notion antérieure de névrose traumatique de guerre, élaborée à partir de la névrose traumatique pensée par Charcot, le maître de Freud. Or Bardamu présente une névrose de guerre romanesque, certes, mais conforme dans sa définition et ses symptômes à la description qu'on trouve dans Au-delà du principe du plaisir, et dans La psychanalyse de la névrose de guerre, ouvrage collectif de Ferenczi, Abraham, Simmel et Jones. "Schreckneurosen" ou "névroses d'effroi" rejoignent les études de la psychiatrie traditionnelle, et seule la notion de conflit intra-narcissique du moi appartient spécifiquement à Freud : «Ce conflit se joue entre l'ancien moi pacifique et le nouveau moi guerrier du soldat, et devient aigu dès que le moi de paix découvre à quel point il court le risque que la vie lui soit retirée à cause des entreprises aventureuses de son double parasite nouvellement formé. [...] Dans les névroses de guerre, ce qui fait peur, c'est bel et bien un ennemi intérieur $»^{20}$. Voyage au bout de la nuit rend compte de l'importance de la pathologie psychique de guerre pour cette génération (qui inclut les surréalistes) et se propose, entre autres, comme la réalisation romanesque d'une thèse psychologique. L'atteste la vision typique du névrosé de guerre dans l'épisode du Stand des Nations: alors que la baraque de tir représente une noce, les cibles

L'hypothèse spéculative d'un principe de destructivité qui dépasse le principe de plaisir est présentée dans $A u$ delà du principe de plaisir en 1920.

${ }^{20}$ Introduction à «La psychanalyse de la névrose de guerre », S. Freud, Résultats, idées, problèmes, Paris, PUF, 1984, p. 245. 
figurent «au premier rang, en zinc, la mariée avec ses fleurs, le cousin, le militaire, le promis. [ ...] Au dernier rang derrière la noce, un autre rang peinturluré, la Mairie avec son drapeau. On devait tirer dans la Mairie aussi quand ça fonctionnait, dans les fenêtres qui s'ouvraient alors d'un coup sec de sonnette, sur le petit drapeau en zinc même on tirait. Et puis sur le régiment qui défilait, en pente, à côté, comme le mien, place Clichy, celui-ci entre les pipes et les petits ballons, sur tout ça on avait tiré tant qu'on avait pu, à présent sur moi on tirait hier, demain» (79-80). Bardamu voit sa propre silhouette parmi les cibles, et il doit tirer sur lui-même pour se massacrer. Il devient l'assassin alter ego de l'assassiné. Son état délirant et hallucinatoire rappelle le vécu traumatique de la guerre. Le traumatisme en soi se situe d'ailleurs dans une ellipse de la narration entre la quatrième section où Bardamu est encore valide et la cinquième où on le retrouve blessé. Le souvenir traumatisant n'est ni narrable ni nommable, et fait effraction par l'hallucination qui saisit Bardamu devant le bien nommé Stand des nations. «Alors je suis tombé malade, fiévreux, rendu fou, qu'ils ont expliqué à l'hôpital, par la peur » (81). Les trois crises postérieures sont liées à la guerre et à la rencontre de Robinson ${ }^{21}$ : pendant la traversée de l'océan où il grelotte de fièvre, au chevet de Bébert malade, et lors de la crise du 4 mai (538) qui réactive la date, fameuse pour les pacifistes, de la première Mutinerie de l'armée française le 4 mai 1917. Bardamu souffre de rêves d'angoisse, de déséquilibres, d'hallucinations visuelles, il est partagé entre son « ancien moi », celui du temps de paix, celui qui croyait au tact, à la délicatesse, parlait et jouait avec de petits allemands, pensait qu'il est défendu de tuer, et un moi de la guerre, celui qui fut encouragé à tuer: «J'avais de la bonne tenue, le maintien modeste, la déférence facile, et la peur toujours de n'être pas à l'heure, et encore le souci de ne jamais passer avant une autre personne dans la vie, de la délicatesse enfin... Quand on est revenu d'un abattoir international en folie, c'est tout de même une référence sous le rapport du tact et de la discrétion » (148). Cette folie de peur panique occupe une place importante dès le début du roman. Elle permet à Céline de questionner l'état des expertises médicales et psychiatriques, et de soupçonner les collisions d'intérêt entre savoir scientifique et pouvoir militaro-politique.

Car pour l'armée, Bardamu est suspect, «ça a fait des histoires » (83). Est-il anarchiste ou syphilitique? Ou encore hystérique ? Son cas est douteux. Le roman est très marqué par la dissimulation de la normalité et la simulation hystérique des symptômes de la névrose de guerre: l'hystérie par la simulation a fait fureur pendant la guerre dans l'espoir d'échapper au combat. A travers le parodique Professeur Bestombes, Céline s'attaque de

\footnotetext{
${ }^{21}$ Le double d'Otto Rank informe peut-être la narration de manière littéraire par l'invention de Robinson.
} 
façon polémique à Gustave Roussy et au «traitement moral des psychonévroses » par la persuasion et l'électricité. Médecin-chef du Centre Neuropsychiatrique de la Dixième Armée à sa création, en avril 1915, Roussy publie en 1917 Les Psychonévroses de guerre et en 1918 Traitement des psychonévroses de guerre. Il dirige l'hôpital Paul Brousse à Villejuif qui se travestit en Bicêtre dans le roman. Le savant Bestombes se réfère aussi à une autre autorité, à Ernest Dupré, «notre grand Dupré [qui] sut établir sa nomenclature désormais célèbre où cette crise [dite d'aveux] figure sous le titre de crise du « rassemblement des souvenirs ». [...] Dupré donne d'autre part, dans sa terminologie si imagée et dont il avait l'apanage, le nom de "diarrhée cogitive de libération" à cette crise » (121). A travers Bestombes, Céline fait précisément allusion à la « psychonévrose émotive » de Dupré. En 1915, Dupré, spécialiste de psychiatrie médicolégale et auteur de Pathologie de l'imagination et de l'émotivité, «triait » les psychopathes à l'hôpital du Val-de-Grâce. D'après les archives des hôpitaux militaires, 50 $\%$ des admissions en hôpital neurologique étaint dues à des manifestations hystériques à partir de 1915, et l'armée s'acharnait à détecter les fraudeurs. D'où la méfiance de Bardamu pour le personnel des médecins militaires aux «grandes oreilles » qui traquent des aveux. Le malade Branledore, lui, a bien compris comment faire passer la simulation de la névrose : en simulant le patriotisme. Le roman loue sa virtuosité histrionesque : le patriotisme du professeur Bestombes le rend aveugle à la comédie, tellement il croit à la vertu thérapeutique de la suggestion patriotique et de la ré-éducation coercitive. Dans un passage hautement carnavalesque, le « reconstituant moral » se confond avec le reconstituant de la morale, c'està-dire avec « de vigoureuses doses d'éthique patriotique, par les véritables injonctions de la morale reconstituante $\gg(124)$.

La satire psychiatrique est concentrée et intensive, mais elle fait partie de la configuration d'ensemble que j'ai d'abord rappelée. Elle est extensible à la science en général, aux Lumières. A l'Aufklärung, à Goethe et à « l'inédite fiction patriotique », puis à la patrie, à la modernité démocratique ${ }^{22}$; «le soldat gratuit ça c'était du nouveau » (93). C'est en effet le peuple qui fait les frais de cette décomposition nationale et de ce « reconstituant (de la) moral(e). Au nom du peuple, Bardamu témoigne en exprimant l'appartenance à une même humanité, plus souffrante qu'agissante puisqu'elle est surtout agie et manipulée par des politiques sans affect, sans cœur ${ }^{23}$. Il manque cruellement un sentir-vrai, un homme qui pense aux hommes et un écrivain qui fonde sur l'affect et sur l'émotion sa vision du peuple et du

\footnotetext{
${ }^{22}$ «C'est une vérité du cœur, la Patrie, comme toutes les vérités essentielles » (123).

${ }^{23}$ Céline reproche en 1933 aux hommes de la gauche d'être des politiques « sans corps », ce qui veut dire aussi sans affect.
} 
rôle qu'on lui fait jouer dans la macabre farce de l'histoire. A ce titre, la misanthropie de Bardamu est à repenser en émotion haineuse pour ce que sont devenus les autres comme le moi, les produits seconds, dénaturés d'une mauvaise socialisation. On est soi-même, issu de la guerre, de cette débandade générale, nationale, morale, converti en "l'ennemi intérieur » au cœur de soi. La haine de soi et des autres pareillement pervertis s'articule à la pitié démocratique. Elle en est la modalité inversée. Si la pitié rousseauiste est un principe fondateur du vivre-ensemble, c'est qu'elle repose sur la réciprocité et la mutualité. «Je ne m'intéresse à lui que pour l'amour de moi », prévient le Second Discours. Mais quand la généralisation de moi à autrui s'opère à partir de la haine et de la honte, que se passe-t-il ? Ou bien la mutualité ne saurait plus qu'être destructive : je ne me désintéresse de lui que par haine de moi. Ou bien il faut originer cette destructivité dans un au-delà du principe de paix et de vie, dans l'horizon de la dislocation, de la déliaison et de la mort. Le propos de Céline ne se déduit pas de l'interprétation rousseauiste et révolutionnaire du bien et du mal ; il prend sa source dans une anthropologie éclairée par la psychanalyse, où la possibilité de l'amour de soi -entendu comme principe de conservation de la vie et peur réciproque de la mort- tient d'un improbable et paradoxal credo : "L'ordure, elle, ne cherche ni à durer ni à croître. Ici, sur ce point, nous sommes bien plus malheureux que la merde, cet enragement à persévérer dans notre état constitue l'incroyable torture. [ ...] Ce corps à nous, travesti de molécules agitées et banales, tout le temps se révolte contre cette farce atroce de durer »» (427).

Céline en conclusion rejoint l'analyse du Contrat social: la civilité négociée et contrôlée par le contrat à l'intérieur d'un état contraste avec la permanence latente de la violence dans les rapports inter-étatiques. En reconnaissant dans la guerre l'actualisation d'une destructivité archaïque, originaire, qu'aucun processus de civilisation ne cultive, n'éradique, il se rapproche de Freud ${ }^{24}$. Rousseau partage d'ailleurs avec Freud l'intuition de la permanence latente de la violence, le risque de la régression, et surtout. Dès juillet 1932, Wilhelm Reich redoutait les conséquences politiques de cette pulsion de mort propicc à tous les conservatismes: « La théorie des pulsions de mort, de la volonté biologique d'autodestruction conduit vers une philosophie de la civilisation que Freud a définie dans son ouvrage, Das Unbehagen in der Kultur, philosophie tendant à prouver que la souffrance humaine est inévitable, puisque les tendances auto-destructrices de l'individu sont indéracinables » (L'analyse caractérielle). Ses craintes se sont avérées justifiées, et le trajet de

\footnotetext{
${ }^{24}$ Rousseau suppose l'homme naturellement pacifique. Il n'y a pas de guerre d'homme à homme mais d'état à état. La guerre ne vise pas l'anéantissement de l'autre, mais elle sert à asseoir le prestige, la maîtrise et la puissance symboliques d'un état ( $\mathrm{cf}$ « L'Etat de guerre », Fragments politiques).
} 
Céline lui-même en confirma la justesse. En tout cas, de Rousseau à Freud et à Reich, le primat de l'affectivité dans les relations politiques ne fait pas le moindre doute.

Reste à savoir comment ces affects, ce sentir-vrai s'exprimeront, comment ce qui se sent obscurément trouvera à se dire fortement et clairement. Pour Céline savoir ce qu'on dit requiert de dire ce qu'on sent : « Cette espèce d'agonie différée, lucide, bien portante, pendant laquelle il est impossible de comprendre autre chose que des vérités absolues, il faut l'avoir endurée pour savoir à jamais ce qu'on dit » (72).

\section{Poétique de l'affectivité}




\section{Le roman de l'affect}

La compassion éprouvée par le lecteur du roman est suscitée par l'identification avec le narrateur Bardamu. L'enjeu du roman à la première personne repose sur la fonction subjective et expressive du discours, qui implique l'énonciation d'un parcours au présent, l'immédiateté jaillissante de la parole. Le romancier cherchait un autre langage, chargé d'émotion immédiate, transmissible mot par mot : «L'émotion ne peut être captée et transcrite qu'à travers le langage parlé... le souvenir du langage parlé ! [...] Mon truc à moi, c'est l'émotif ! » ${ }^{25}$. Le champ lexical de la parole a d'ailleurs le premier et le dernier mot : «Moi j'avais jamais rien dit ...Qu'on n'en parle plus ». L' attentat oral contre les codes culturels, la «belle langue » dans l'espace sacré de la littérature est donc un scandale rhétorique et poétique. Céline travaille à l'efficacité mordante de la parole, au parler vif et incisif. La circulation de la parole, c'est l'affaire des concierges, du peuple, qui donnent à la vi(ll)e des histoires, du piment. Il existe une virtuosité de l'éloquence a-rhétorique dont Madame Henrouille est un modèle, comme aussi les concierges. Dans leur médisance, en outre, il y a un art de dire qui "mord, vulnère, incise, tracasse, obsède" (271), art de dire dont l'effet quasi corporel et affectif fonde le monde commun et sensible du partage des émotions.

Comme la seule fonction subjective serait insuffisante à produire ce résultat, la fonction conative vient la soutenir. Le témoignage s'adresse, dès lors qu'on témoigne de et pour. Il n'existe pas de pitié sans la pensée d'un nous préalable : «Il fallait bien être quelque part cependant en attendant le matin, quelque part dans la nuit. On ne pouvait pas éviter tout. Depuis ce temps-là, je sais ce que doivent éprouver les lapins en garenne.

Ça vient drôlement la pitié. [...] La grande défaite, en tout, c'est d'oublier, et surtout ce qui vous a fait crever [...]. Quand on sera au bord du trou faudra pas faire les malins nous autres, mais faudra pas oublier non plus, faudra raconter tout sans changer un mot de ce qu'on a vu de plus vicieux chez les hommes et puis poser sa chique et puis descendre. Ça suffit comme boulot pour une vie tout entière » (38). Le «nous » se constitue du partage de la panique de la mort, le «vous » explétif (« ce qui vous a fait crever») intègre le narrataire dans cette communauté morbide. Bardamu répète sa responsabilité d'être parlant et communiquant: «je dirai tout un jour si je peux vivre assez longtemps pour tout raconter » (311), responsabilité accrue par l'invraisemblance de la fable qu'il conte. Même et surtout si les lecteurs ne peuvent y croire, il faut dire : «il s'est passé des choses et des choses qu'il est pas facile de raconter à présent, à cause que ceux d'aujourd'hui ne les comprendraient déjà plus » (66). Des

\footnotetext{
${ }^{25}$ Entretiens avec le Professeur Y., Paris, Gallimard, 1955, reéd. 1983, p. 28-40.
} 
attestations de réalité, de vérité et de sincérité abondent donc dans les interpellations au lecteur: «Je vous le dis moi. J'ai essayé. C'est pas la peine » (268); «Avouons-le » (271); « Ne l'oublions jamais [...] Je vous le dis » (272), transformant la lecture en responsabilité pragmatique et éthique.

Outre l'effet de proximité et d'intimité, l'effet de voix suscité par le réalisme rythmique suggère une véridicité, une authenticité ontologique. La voix, c'est ce que les blancs ne peuvent truquer; elle reste agressive. De même l'accent distingué, «l'accent qui a un petit fouet dedans »(507) sonne effilé comme s'il allait «trancher». La voix est sensible et demande d'être sentie. Il faut sentir comme un animal, non pas croire aux mots : « La chienne au moins ne croit que ce qu'elle sent » (124). L'esthétique célinienne rejoint une éthique de la naïveté sans angélisme, de l'ingénuité animale, et la posture testimoniale se fonde sur ce nous de la compassion démocratique, dans quel le lecteur est invité à se compter.

La parole vive présente le foisonnement, le jet du réel sautant à la gorge de la conscience et de l'expression. Le verbe polémique et politique tord le coup à la syntaxe, à la langue de l'Académie française. D'où la parataxe, la juxtaposition, la suspension, les ellipses, les réticences et les redondances, soit un excès et un défaut de langue. La « langue de rappel » de la phrase segmentée, typique de Céline, invente un nouveau réalisme verbal qui rompt avec les codes de l'écriture romanesque mimétique, c'est-à-dire avec une écriture qui pour représenter le monde le rend intelligible, logique, présentable. Ainsi la parole drue de Bardamu agresse à côté de l' «héroïsme mutin» de Musyne qui s'exerce à «la poésie héroïque » dans un «petit répertoire très coquet d'incidents de guerre » produit-il un sfumato aussi esthétisant que trompeur : «Elle possédait le don de mettre ses trouvailles dans un certain lointain dramatique. [...] Il faut croire Claude Lorrain, les premiers plans d'un tableau sont toujours répugnants et l'art exige qu'on situe l'intérêt de l'œuvre dans les lointains, dans l'insaisissable, là où se réfugie le mensonge, ce rêve pris sur le fait, et seul amour des hommes » (107). En face, les «grossiers bobards » de Bardamu opèrent une distanciation critique, désaliènent l'auditeur par la force de provocation du proche et du dégoûtant.

La haine de la rhétorique rejoint donc une honte éthique. Cette haine se manifeste par le discours rapporté, réduit à une énumération de stéréotypes politiques de la langue de bois, figée, prévisible. Par exemple le discours de "l'intérêt général" du maire de Noirceur et le morceau d'éloquence du médecin chef sont parodiques. Personnifiant et glorifiant la France, à coups d'hyperboles, de superlatifs, ils s'achèvent par l'appel à la vertu sacrée à l'égard du sol chéri, c'est-à-dire par un couplet barrésien et mystique de patriotisme promu spirituel. La valeur du sol et du sang versé pour lui relève de la rhétorique belliciste qui a déclenché et 
soutenu les années de guerre. Bardamu y réagit par une peur panique: « J'étais devenu, devant tout héroïsme verbal ou réel, phobiquement rébarbatif ».

\section{L'écriture et la parole : la responsabilité de l'écrivain dans la démocratie}

L'éloquence disloquée de l'écriture et la dissonance pamphlétaire de Voyage au bout de la nuit manifestent l'implication émotionnelle des partenaires de l'œuvre (auteur, narrateur, lecteur) et la crise de la démocratie. Car outre le style oral et émotif, il faut en relever la «politique de la narration ». Nelly Wolf, à qui nous empruntons l'expression rappelle dans Le Roman de la démocratie ${ }^{26}$ que le roman est le genre de la démocratie parce qu'il se fonde sur le contrat entre narrateur et narrataire, et parce qu'il met en scène les options différentes, voire divergentes, le conflit et le débat associés à tout espace démocratique. Non seulement il se développe à partir du dix-huitième siècle dans l'horizon de la pensée et des valeurs démocratiques, mais il est par la langue en accord ou en désaccord avec un projet démocratique. Dans le roman à la troisième personne, dominant au cours du dix-neuvième siècle, trois traitements de la langue du peuple, trois solutions linguistiques se présentent. Soit la parlure populaire est dominée par la voix narrative (comme dans Graziella de Lamartine ou Jude the obscure de Thomas Hardy). Soit la langue claire, intelligible du narrateur du récit à la troisième personne concorde avec l'idéal communicationnel de la démocratie et seules les paroles des personnages introduisent de l'hétérogène (Dickens, Balzac, Le Feu de Barbusse). Soit enfin la langue du narrateur est contaminée par le parler vif et hétérogène (Eugène le Roy: Jacquou le croquant, et La trilogie de Jacques Vingtras de Vallès). Les romans d'éducation au dix-neuvième siècle exposaient le conflit de l'individu et de la société, exhibaient l'acte d'association (contrat) et les ratés de la socialisation volontaire (Sorel, Chardon-Rubempré, Moreau). Au vingtième siècle, ils représentent les maladies du contrat, à la première personne, de telle sorte que dans le pacte de lecture le lecteur se reconnaisse en chacun des semblables de cette « communauté malade des ayants droit ».

La maladie de la communauté s'avoue dans le récit à la première personne. Que signifie en effet le renoncement au narrateur, sinon la crise de l'autorité ? Celle-ci se double de la crise des garanties politiques depuis la guerre de 14, qui se manifeste dans la polyphonie

\footnotetext{
${ }^{26}$ N. Wolf, Le Roman de la démocratie, Presses Universitaires de Vincennes, 2003.
} 
de discours anciennement dits d'autorité (politique, militaire, scientifique, économique) désormais réduits à des opinions subjectives et contingentes ${ }^{27}$.

Quand le contrat de la troisième personne et du narrateur impersonnel est annulé au profit d'une parole urgente, pulsionnelle, qui dit la crise de toute autorité par le conflit entre la langue vulgaire et la langue scolaire, quand disparaît l'autorité fictive, le roman de formation se lit comme un roman du contrat qui met en scène la crise du contrat, crise telle que l'entrée en société ne se fait pas ${ }^{28}$. Le narrataire confident des aventures noctambulesques de Bardamu, comme alter ego compatissant par identification avec le narrateur se vit comme méritant, à son tour, compassion.

La vocifération de Céline se fait l'écho de ceux qui n'ont pas la parole, elle exerce la responsabilité civique par les moyens de l'écrivain, l'écriture d'une oralité. Barthes, dans $L e$ degré Zéro de l'écriture, met en regard «L'écriture et la parole » : «Pendant ces moments où l'écrivain suit les langages réellement parlés, non plus à titre pittoresque, mais comme les objets essentiels qui épuisent tout le contenu de la société, l'écriture prend pour lieu de ses réflexes la parole réelle des hommes.[...] Dans l'oeuvre de Céline par exemple, l'écriture n'est pas au service d'une pensée, comme un décor réaliste réussi, qui serait juxtaposé à la peinture d'une sous-classe sociale, elle représente vraiment la plongée de l'écrivain dans l'opacité poisseuse de la condition qu'il décrit. [...] L'appréhension d'un langage réel est pour l'écrivain l'acte littéraire le plus humain. [...] On voit se dessiner par là l'aire possible d'un nouvel humanisme: à la suspicion générale qui atteint le langage tout au long de la littérature moderne, se substitue une réconciliation du verbe de l'écrivain et du verbe des hommes. C'est seulement alors que l'écrivain pourrait se dire entièrement engagé, lorsque sa liberté poétique se placerait à l'intérieur d'une condition verbale dont les limites seraient celles de la société, et non celles d'une convention ou d'un public ».

A un moment où le nom de Céline court sous les plumes d'un Houellebecq, d'un Philippe Muray, voire d'un Maurice G. Dantec, il importe de veiller à se souvenir qu'écrire Voyage au bout de la nuit fut pour l'auteur un moyen de prendre ses responsabilités, de « ne

\footnotetext{
${ }^{27}$ « Avec la première guerre mondiale, l'identité narrative de la démocratie et sa capacité à se mettre en fiction semblent donc gravement compromises. Ce n'est pas simplement, comme le dit l'histoire littéraire, qu'après les horreurs de la guerre, on ne peut plus raconter comme avant. Le danger est que la faculté narrative soit atteinte. En ce sens le déni de fiction effectué par Virginia Woolf résonne comme un avertissement». N. Wolf, op. cit., p. 157. Le déni de fiction dont elle parle renvoie à la construction fracturée de La Promenade au phare, dont la seconde partie correspond au temps de la guerre qui est traitée comme une parenthèse : le titre "Time passes » évoque un vide événementiel.

${ }^{28}$ L'inverse se vérifiait dans Le roman de l'énergie nationale de Barrès, "roman idéologique » où l'instance narratoriale est autoritaire et impose le refus idéologique de la démocratie (1894-1907: nationalisme, antiparlementarisme, antisémitisme, dénonciation de l'école républicaine, culte du soldat et de la terre.
} 
pas déserter» et de «penser aux hommes ». Les mots de François Bon sur la crise démocratique de notre siècle qui ouvrent Daewoo, dans leur retenue et leur colère, font retentir un peu de cette rage de dire, de cette responsabilité civique et de cette espérance en la littérature que Céline incarne :

« Refuser. Faire face à l'effacement même.

Pourquoi appeler roman un livre quand on voudrait qu'il émane de cette présence si étonnante parfois de toutes choses, devant un portail ouvert mais qu'on ne peut franchir, le silence approximatif des bords de ville un instant tenu à distance, et que la nudité crue de cet endroit précis du monde on voudrait qu'elle sauve ce que béton et ciment ici enclosent, pour vous qui n'êtes là qu'en passager, en témoin ? [ ...] Croire que la vieille magie de raconter des histoires, si cela ne change rien à ce qui demeure [...] vous permettrait d'honorer jusqu'en ce lieu cette si vieille tension des choses qui se taisent et des mots qui les cherchent, tandis que vous voudriez pour vous-même qu'un peu de solidité ou de sens encore en provienne?»

\section{Marie-Hélène BOBLET}

Maître de conférences en littérature française, Université Paris III- Sorbonne nouvelle 